\title{
Placental Fractalkine Is Up-Regulated in Severe Early-Onset Preeclampsia
}

\author{
Monika Siwetz, ${ }^{*}$ Martina Dieber-Rotheneder, ${ }^{\dagger \dagger}$ Mila Cervar-Zivkovic, ${ }^{\dagger}$ Daniel Kummer, ${ }^{*}$ Julia Kremshofer, ${ }^{*}$ Gregor Weiss, * \\ Florian Herse, ${ }^{\S}$ Berthold Huppertz, ${ }^{*}$ and Martin Gauster*
}

From the Institutes of Cell Biology, Histology and Embryology* and Pathology ${ }^{\ddagger}$ and the Department of Obstetrics and Gynaecology, ${ }^{\dagger}$ Medical University Graz, Graz, Austria; and the Experimental and Clinical Research Center, ${ }^{\S}$ a Joint Cooperation between the Charite Medical Faculty and the Max-Delbrueck Center for Molecular Medicine, Berlin, Germany

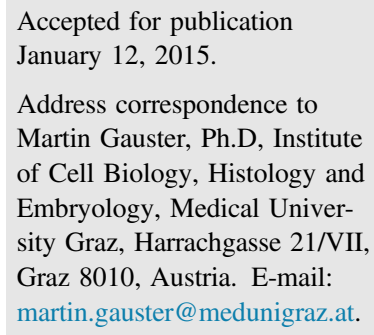

Although detailed processes underlying the etiology and pathophysiology of preeclampsia (PE) are still not completely understood, the release of placental factors into the maternal circulation is one of the hallmarks of the syndrome. ${ }^{1,2}$ These placental factors are suggested to include not only placental debris, but also a host of molecules, such as angiogenic factors, components of the renin-angiotensin system, and inflammatory cytokines, ${ }^{3-5}$ which may trigger maternal vascular dysfunction and an increased maternal inflammatory response. Recently, the chemokine fractalkine [or chemokine (C-X3-C motif) ligand 1, CX3CL1] was shown to be expressed at the apical microvillous plasma membrane of the syncytiotrophoblast in human placenta, from where it is released into the maternal circulation by constitutive metalloprotease-dependent shedding. ${ }^{6}$ According to previous placental explant studies, the release of placental fractalkine significantly increases from first trimester toward term of pregnancy. ${ }^{6}$ In general, fractalkine, together with another chemokine termed CXCL16, represents a unique group of chemokines, because they are not expressed as soluble chemokines but as transmembrane multidomain molecules consisting of a chemokine domain, a mucin-like stalk, a transmembrane segment, and a short cytoplasmic tail. ${ }^{7-9}$ The extracellular domains, representing the chemokine domain and the mucin-like stalk, can be shed by the a disintegrin-like metalloproteinases (ADAMs) 10 and 17 into a soluble form. ${ }^{10-12}$ On the basis of these observations, fractalkine exists as membrane-bound and soluble forms, both of which bear different functions. Although the membrane-bound form

Supported by the Austrian Science Fund grant P23859-B19 (M.G.) and the Deutsche Forschungsgemeinschaft grant HE6249/1-1 (F.H.).

Disclosures: None declared. 
promotes flow-resistant adhesion of leukocytes to endothelial or epithelial cells via its corresponding $\mathrm{G}$ protein-coupled, seven-transmembrane receptor CX3C chemokine receptor 1 (CX3CR1), the soluble form has chemoattractive activity for monocytes, natural killer cells, and T cells. ${ }^{13}$ Attention to this chemokine-receptor duo is constantly increasing, and it is suggested to be involved in the pathogenesis of various inflammatory disorders, such as Crohn disease, allergic asthma, rheumatoid arthritis, and atherosclerosis. ${ }^{14}$

Knowledge regarding a role of fractalkine in human pregnancy is, albeit extending in recent years, restricted to a few studies. Initial experiments with trophoblast cell lines suggest fractalkine to be involved in establishing first contact between the blastocyst and the uterine epithelium, and to promote trophoblast migration through the maternal decidua. ${ }^{15-17}$ Another study suggests a role of fractalkine in placental microvasculature remodeling, because the up-regulation of placental fractalkine was associated with increased fetal microvessel density in placental villi from pregnancies complicated by diabetes mellitus. ${ }^{18}$ Moreover, increased release of soluble fractalkine was determined in perfusion fluids of perfused placental lobules in response to lipopolysaccharide and hypoxia, ${ }^{19}$ indicating up-regulation of fractalkine in the placental endothelium under proinflammatory conditions. Recently, increased fractalkine expression was shown in human amniotic epithelial cells isolated from pregnancies complicated by chorioamnionitis. ${ }^{20}$

Taken together, available data suggest up-regulation of fractalkine in the uteroplacental unit in response to inflammatory conditions. Indeed, normal pregnancy is accompanied by a mild systemic inflammatory response, which strengthens during pregnancy and peaks during the third trimester. ${ }^{21,22}$ This mild systemic inflammatory response is characterized by increased circulating inflammatory cytokines and the activation of lymphocytes, granulocytes, and monocytes. ${ }^{23,24}$ In PE, a maternal syndrome of hypertension and proteinuria, the intensity of the systemic inflammatory response is more vigorous, including significantly increased proinflammatory maternal circulating cytokines, when compared to normotensive pregnancies. ${ }^{2}$ Thus, the hypothesis was tested whether placental fractalkine is up-regulated in severe early-onset PE and, moreover, whether exogenously applied proinflammatory cytokines tumor necrosis factor (TNF)- $\alpha$ and IL-6, mimicking proinflammatory maternal circulating cytokines, are able to increase fractalkine expression and release in placental villous explant culture.

\section{Materials and Methods}

\section{Human Placenta Tissue Samples}

The study was approved by the ethical committee of the Medical University of Graz (Graz, Austria), and informed consent was obtained from the women. Placentas were obtained immediately after caesarean section from women with singleton pregnancies complicated with severe early-onset
Table 1 Characteristics of the Study Group

\begin{tabular}{lcc}
\hline Characteristics & Control & $\begin{array}{l}\text { Severe } \\
\text { early-onset PE }\end{array}$ \\
\hline No. of subjects & 7 & 8 \\
Maternal age (years) & $30.57 \pm 6.58$ & $31.50 \pm 6.57$ \\
BMI $\left(\mathrm{kg} / \mathrm{m}^{2}\right)$ & $21.43 \pm 3.61$ & $25.01 \pm 3.80$ \\
Gestational age (weeks) & $31.43 \pm 3.10$ & $32.13 \pm 1.73$ \\
$\begin{array}{l}\text { Weight of placenta }(\mathrm{g}) \\
\text { Weight of neonate }(\mathrm{g})\end{array}$ & $352.86 \pm 127.24$ & $348.57 \pm 112.61$ \\
$\begin{array}{l}\text { Percentile of } \\
\quad \text { neonate weight }\end{array}$ & $45.43 \pm 30.48$ & $18.88 \pm 7.86^{*}$ \\
$\begin{array}{l}\text { Mode of delivery } \\
\quad(\mathrm{CS} / \text { vaginal ratio) }\end{array}$ & $7: 0$ & $8: 0$ \\
\hline
\end{tabular}

Data are presented as means \pm SD unless otherwise indicated.

${ }^{*} P<0.05$.

BMI, body mass index; CS, caesarean section; $\mathrm{PE}$, preeclampsia.

PE. The control group comprised gestational age-matched placentas, delivered by caesarean section because of placenta previa with vaginal bleeding or cervical insufficiency from women who had no clinical evidence for infection. Severe early-onset PE was defined as hypertension ( $>160 / 110$ $\mathrm{mmHg}$ ) plus mild proteinuria or mild hypertension plus severe proteinuria ( $\geq 5 \mathrm{~g}$ protein/24-hour urine collection) with delivery at $<34$ weeks' gestation. Characteristics of the study population are shown in Table 1. Pieces of approximately $0.5 \mathrm{~g}$ moist mass were dissected from the central part of the placenta near the umbilical cord, washed in cold buffered saline, snap frozen in liquid nitrogen, and stored for subsequent analysis. Pregnancies complicated by clinical evidence of infection, steroid treatment, AIDS, alcohol abuse, and/or drug abuse were excluded. First-trimester placenta tissues ( $n=20$; mean gestational age, $9.9 \pm 1.8$ weeks) were obtained from women (mean maternal age, $29.6 \pm 8.0$ years; mean body mass index, $24.8 \pm 4.9$ ) undergoing pregnancy terminations for psychosocial reasons.

\section{Gene Expression Analysis}

Total RNA from placental tissue was isolated with the peqGOLD TriFast reagent (Peqlab, Erlangen, Germany) and a tissue homogenizer (IKA TA10 basic, Ultra-Turrax; IKA Werke GmbH \& Co. KG, Staufen, Germany), according to the manufacturer's protocol. After quality check, $2 \mu \mathrm{g}$ total RNA was reverse transcribed to cDNA, using the High-Capacity cDNA Reverse Transcription Kit (Applied Biosystems, Foster City, CA). cDNA was subsequently subjected to quantitative PCR using TaqMan Gene Expression Assays for fractalkine (CX3CL1, Hs00171086_m1), ADAM10 (Hs00153853_m1), ADAM17 (Hs01041915_m1), and ribosomal protein L30 (Hs00265497_m1) and the TaqMan Universal PCR Mastermix (Applied Biosystems). cDNA and kit components were mixed in $20 \mu \mathrm{L}$ total volume per well (96-well plates; Roche, Mannheim, Germany), according to the manufacturer's instructions, and amplified using a Bio-Rad CFX96 Real-Time PCR System (Bio-Rad Laboratories GmbH, 
Vienna, Austria). Cycle threshold values were automatically generated by the CFX Manager software version 3.1 (Bio-Rad), and relative quantification of gene expression was calculated by standard $\Delta \mathrm{Ct}$ method using the expression of ribosomal protein L30 as reference. Data are presented as mean of $2^{-\Delta \mathrm{Ct}}$ values.

\section{Fractalkine ELISA}

Fractalkine was measured in placental tissue homogenates and conditioned culture medium of placental explant culture using a quantitative sandwich enzyme immunoassay [Human CX3CL1/Fractalkine Quantikine Enzyme-Linked Immunosorbent Assay (ELISA); R\&D Systems Europe Ltd., Abingdon, UK]. Conditioned culture media from placental explant culture were centrifuged at $1500 \times g$ and $4^{\circ} \mathrm{C}$ for 5 minutes. Placental tissue homogenates were obtained by homogenizing placental explants or placental tissue specimens in radioimmunoprecipitation assay (RIPA) buffer (Sigma-Aldrich, St. Louis, MO), including Protease Inhibitor Cocktail (Roche) and centrifugation at $8000 \times g$ and $4^{\circ} \mathrm{C}$ for 10 minutes. After centrifugation, $100 \mu \mathrm{L}$ of clear supernatants from conditioned culture media or placental tissue homogenates was subjected to immunoassays, according to the manufacturer's instruction. Complete culture medium incubated without explants and RIPA buffer served as blanks for fractalkine measurement in conditioned supernatants and tissue homogenates, respectively. Samples were measured in duplicate, and obtained fractalkine concentrations were normalized to total tissue protein, which was determined in homogenates according to the Lowry method.

\section{Immunohistochemistry}

Formalin-fixed, paraffin-embedded placental tissue sections (5 $\mu \mathrm{m}$ thick) from six severe early-onset PE cases and six gestational age-matched controls were mounted onto Superfrost Plus slides (Menzel/Thermo Fisher Scientific; Gerhard Menzel GmbH, Braunschweig, Germany). A standard deparaffinization procedure was followed by boiling slides in Epitope Retrieval Solution, pH 9.0 (Novocostra, Leica Biosystems Newcastle Ltd., Newcastle upon Tyne, UK) for 7 minutes at $120^{\circ} \mathrm{C}$ in a decloaking chamber (Biocare Medical, Concord CA). Sections were immunostained using a staining robot (Autostainer 360; Thermo Fisher Scientific) and the UltraVision Large Volume Detection System HRP Polymer Kit (Thermo Fisher Scientific), as previously described. ${ }^{6}$ In brief, endogenous peroxidase was blocked using the hydrogen peroxidase block for 10 minutes. After three washing steps with Tris-buffered saline, including $0.05 \%$ Tween 20 (Merck KGaA, Darmstadt, Germany), background blocking was performed using Ultra Vision Protein Block (Thermo Fisher Scientific) for 5 minutes. Monoclonal anti-human CX3CL1/ fractalkine antibody (clone 81513; R\&D Systems) was diluted $1: 1000(0.5 \mu \mathrm{g} / \mathrm{mL}$ working concentration $)$ in Antibody Diluent (Dako North America Inc., Carpinteria, CA) and
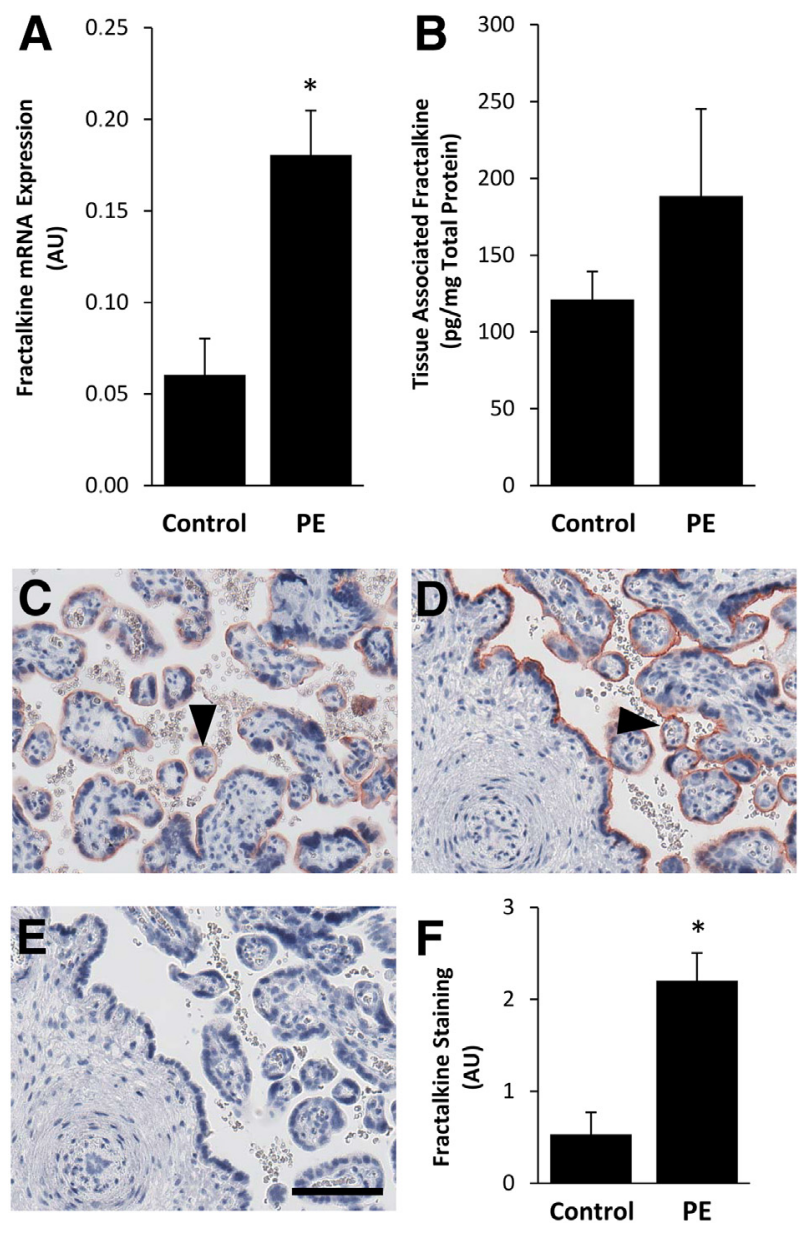

Figure 1 Placental fractalkine is up-regulated in severe early-onset preeclampsia (PE). A: Quantitative PCR analysis shows significantly increased fractalkine expression in placental tissues from severe early-onset $\mathrm{PE}$, compared to gestational age-matched controls. B: Enzyme-linked immunosorbent assay analysis shows increased fractalkine protein levels in placental tissue homogenates from severe early-onset PE, compared to gestational age-matched controls. Immunohistochemistry of placental tissue sections from gestational age-matched controls (C) and severe earlyonset PE (D) show fractalkine localization at the syncytiotrophoblast (arrowheads), where staining is more pronounced in severe early-onset PE. E: Incubation with negative control mouse IgG reveals no staining. F: Quantification of immunohistochemistry shows significantly increased fractalkine staining in placental tissue sections from severe early-onset PE $(n=6)$, compared to gestational age-matched controls $(n=6)$. Values are given as means $\pm \operatorname{SEM}(\mathbf{A}, \mathbf{B}$, and $\mathbf{F}) . n=8$ (A, PE group); $n=7$ (A, control group); $n=6\left(\mathbf{F}, \mathrm{PE}\right.$ and control groups). ${ }^{*} P \leq 0.05$. Scale bar $=100 \mu \mathrm{m}(\mathbf{C}-\mathbf{E})$. $A U$, arbitrary unit.

incubated on slides for 45 minutes at room temperature. After three Tris-buffered saline, including $0.05 \%$ Tween 20, washing steps, Primary Antibody Enhancer (Thermo Fisher Scientific) was applied to slides for 10 minutes at room temperature. Thereafter, slides were washed and detection was achieved by incubation with the anti-mouse/rabbit UltraVision HRP-labeled polymer system (15 minutes) and 3-amino-9ethylcarbacole (Thermo Fisher Scientific), according to the manufacturer's instructions. Nuclei were stained with hemalaun, and slides were mounted with Kaiser's glycerol gelatin. 
For negative controls, slides were incubated with negative control mouse IgG1 (Dako) at the same concentration as mentioned above and did not show any staining. Preadsorption of monoclonal anti-human CX3CL1/fractalkine antibody with recombinant human full-length fractalkine has previously been described and confirmed specificity of the used antibody. ${ }^{6}$

\section{Quantification of Immunohistochemical Fractalkine Staining}

For unbiased quantification of above-described placental fractalkine staining, a microscope (model DM6000B; Leica) equipped with a motorized stage, a digital camera (model DP72; Olympus Austria GmbH, Vienna, Austria), and the newCAST stereology software version 4.5.1.324 (Visiopharm, Hoersholm, Denmark) was used for acquisition of six systematically randomly selected images per slide. To evaluate the acquired images, a pixel classification method using the ilastik software version 1.1.3 (http:// ilastik.org/download.html, last accessed November 11, $2014)^{25}$ was applied to classify the pixels of the images into different object classes (stained and unstained). The features of one representative image were used to define pixel classes on the basis of the differences in brightness, color, and texture. The generated classification features were applied to all images of both groups. The segmentations were then exported as png graphics and analyzed by counting the pixels for each image using the EBImage package version 3.0 (Bioconductor, Seattle, WA; http:// www.bioconductor.org) and $\mathrm{R}$ statistics (http://www.rproject.org). Obtained values were related to villous surface, which was determined in corresponding images using the Zeiss AxioVision software version 4.8.2.0 (Carl Zeiss, $\mathrm{GmbH}$, Vienna, Austria).

\section{Placental Explant Culture}

For placental explant culture, villous tissues from human first trimester $(n=20$, between gestational weeks 8 and 12) were washed thoroughly in buffered saline and dissected into small pieces of approximately $5 \mathrm{mg}$ moist mass. Placental explants were cultured in 12-well dishes (Nunc; Thermo Fisher Scientific) and $2 \mathrm{~mL} /$ well Dulbecco's modified Eagle's medium/F12 (dilution 1:1; Gibco; Life Technologies-Thermo Fisher Scientific) supplemented with $10 \%$ fetal calf serum, penicillin/streptomycin, amphotericin $\mathrm{B}$, and L-glutamine in a hypoxic workstation (BioSpherix Ltd., Lacona, NY) under $2.5 \%$ oxygen at $37^{\circ} \mathrm{C}$ for indicated time points. For cytokine treatments, complete culture medium was supplemented with recombinant human IL-6 (Peprotech, Rocky Hill, NJ) and/or recombinant human TNF- $\alpha$ (Peprotech) at indicated working concentrations. For salicylate experiments, culture medium was supplemented with either TNF- $\alpha$ alone or TNF- $\alpha$ in combination with sodium salicylate (Sigma-Aldrich, Vienna, Austria) at

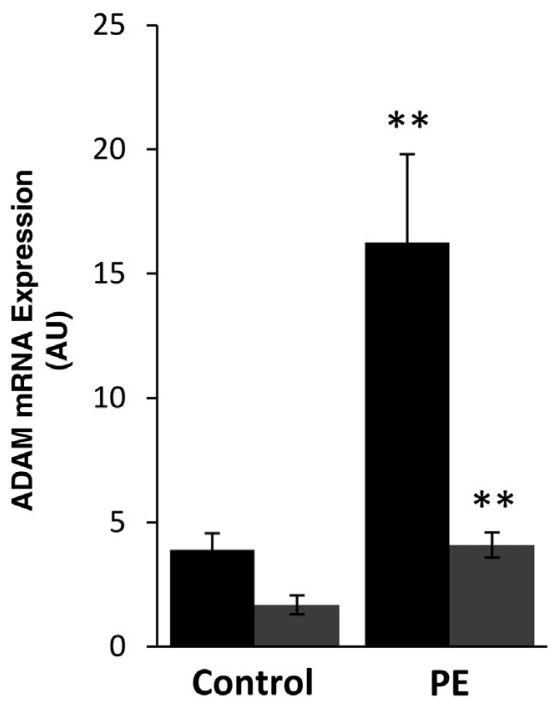

Figure 2 A disintegrin and metalloproteases (ADAMs) 10 and 17 are up-regulated in severe early-onset preeclampsia (PE). Quantitative PCR analysis shows significantly increased expression of ADAM10 (black bars) and ADAM17 (gray bars) in placentas from severe early-onset PE, compared to controls. Values are given as means \pm SEM. $n=8$ (PE group); $n=7$ (control group). ${ }^{* * P} \leq 0.01$. AU, arbitrary unit.

indicated concentrations. Cultivation of explants in complete culture medium without cytokines served as a control. After incubation, conditioned culture media were collected and placental explants were homogenized in RIPA buffer, including protease inhibitor cocktail using a tissue homogenizer.

\section{Analysis of Placental Explant Viability}

Effects of treatments on viability of placental explants were evaluated after culture by measurement of released lactate dehydrogenase (LDH) activity in culture supernatants using LDH cytotoxicity detection kit (Takara Bio Inc., eubio, Vienna, Austria), according to the manufacturer's protocol. Obtained absorbance values were normalized to total protein of respective explant homogenates.

\section{Western Blot Analysis}

Placental villous explants were washed with phosphatebuffered saline and homogenized in RIPA buffer, including protease inhibitor cocktail. Protein concentration was determined according to Lowry method, and $40 \mu \mathrm{g}$ total protein was separated on precast $10 \%$ Bis-Tris gels (NuPAGE, Novex; Life Technologies). After electrophoresis, protein was semidry blotted on $0.45 \mu \mathrm{m}$ nitrocellulose membrane (Hybond; GE Healthcare Europe GmbH, Vienna, Austria) and blotting efficiency was determined by staining membranes with Ponceau $\mathrm{S}$ solution (Sigma-Aldrich, St. Louis, MO). Immunodetection was performed with a chemiluminescent immunodetection kit (Western Breeze; Life Technologies), according to the 


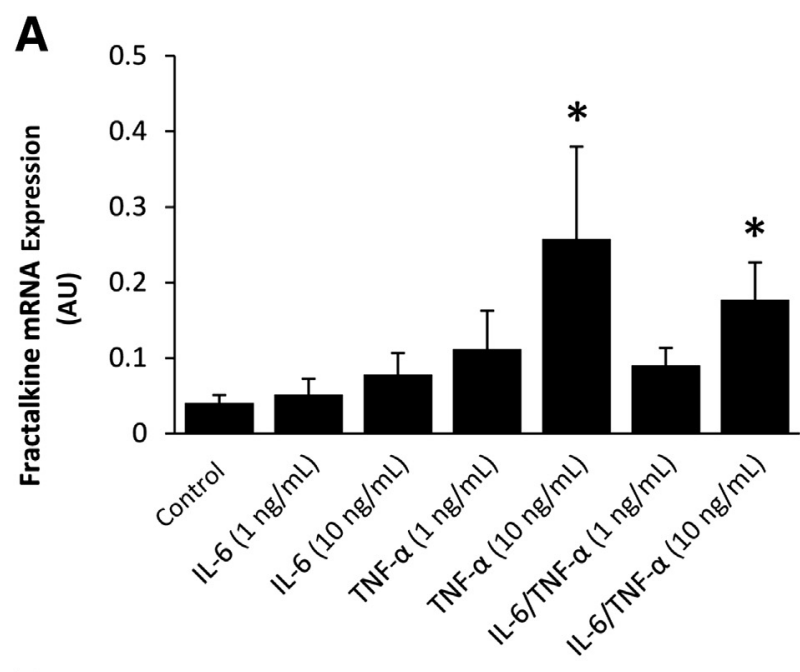

B

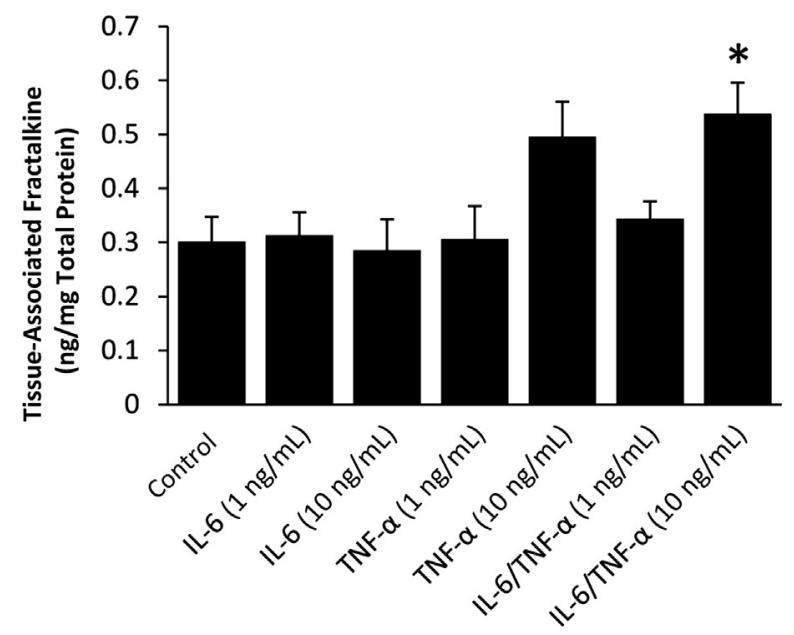

C

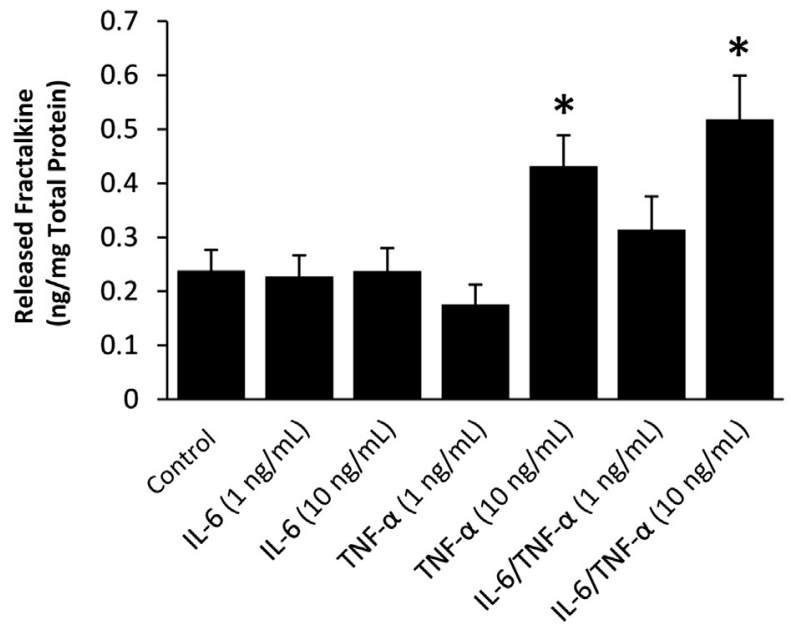

Figure 3 Placental fractalkine is up-regulated by tumor necrosis factor (TNF)- $\alpha$, but not IL- 6 . Placental explants $(n=8)$ were incubated with indicated concentrations of TNF- $\alpha$ and/or IL- 6 for 48 hours. Quantitative PCR analysis (A) and enzyme-linked immunosorbent assay analysis of tissue homogenates (B) and culture supernatants (C) consistently show increased fractalkine expression and secretion only in the presence of TNF- $\alpha$ and TNF- $\alpha$ in combination with IL- 6 at concentrations of $10 \mathrm{ng} / \mathrm{mL}$. IL- 6 alone has no effect. Values are given as means $\pm \mathrm{SEM}$. ${ }^{*} P \leq 0.05$. AU, arbitrary unit. manufacturer's instructions. Rabbit monoclonal anti-NF- $\mathrm{B}$ p65 antibody (clone C22B4; Cell Signaling, Danvers, MA) and rabbit monoclonal anti-phospho-NF- $\mathrm{B}$ p65 (clone 93H1; Cell Signaling) were diluted in blocking solution 1:1000 and incubated on membranes overnight at $4^{\circ} \mathrm{C}$. Images were acquired with FluorChem Q System (Alpha Innotech, Cell Biosciences, Santa Clara, CA), and band densities were analyzed with Alpha View SA software version 3.4.0 (Alpha Innotech, Cell Biosciences).

\section{Statistical Analysis}

Data were analyzed using SigmaPlot software version 12.5 (Systat Software Inc., San Jose, CA) and are presented as means \pm SEM. Data were subjected to normality test (Shapiro-Wilk test) and equal variance test. In case of normally distributed data, differences between groups were tested using a two-tailed $t$-test. Otherwise, a Mann-Whitney rank sum test was applied. For multiple-comparison procedure, one-way repeated measures analysis of variance was followed by Holm-Sidak method to isolate groups that differ from the others. $P<0.05$ was considered statistically significant.

\section{Results}

\section{Characteristics of Patients}

There were no significant differences between patients in terms of maternal age, gestational age, body mass index, mean placental weight, and mean birth weight, whereas the mean percentile of neonate weight was significantly lower in the severe early-onset PE group (Table 1).

\section{Placental Fractalkine Expression Is Increased in Severe Early-Onset PE}

Quantitative gene expression analysis showed $2.99 \pm$ 0.40-fold increased fractalkine expression in placentas from severe early-onset PE, when compared to gestational agematched controls (Figure 1A). ELISA analysis of tissueassociated fractalkine protein levels indicated the same trend and showed $1.55 \pm 0.47$-fold increase in placental tissue from severe early-onset PE, which, however, did not reach statistical significance (Figure 1B). Immunohistochemistry of placental tissues from control (Figure 1C) and severe early-onset PE (Figure 1D) localized fractalkine expression in the syncytiotrophoblast, where staining was more pronounced in severe early-onset PE, compared to controls. Incubation with negative control mouse IgG revealed no staining (Figure $1 \mathrm{E}$ ). Although the analysis of tissue-associated fractalkine protein by ELISA only showed a trend of increase in severe early-onset PE, quantification of immunohistochemical staining for fractalkine by a softwarebased pixel classification approach revealed $4.16 \pm 0.56$-fold increased staining in placental tissue sections from severe 
A
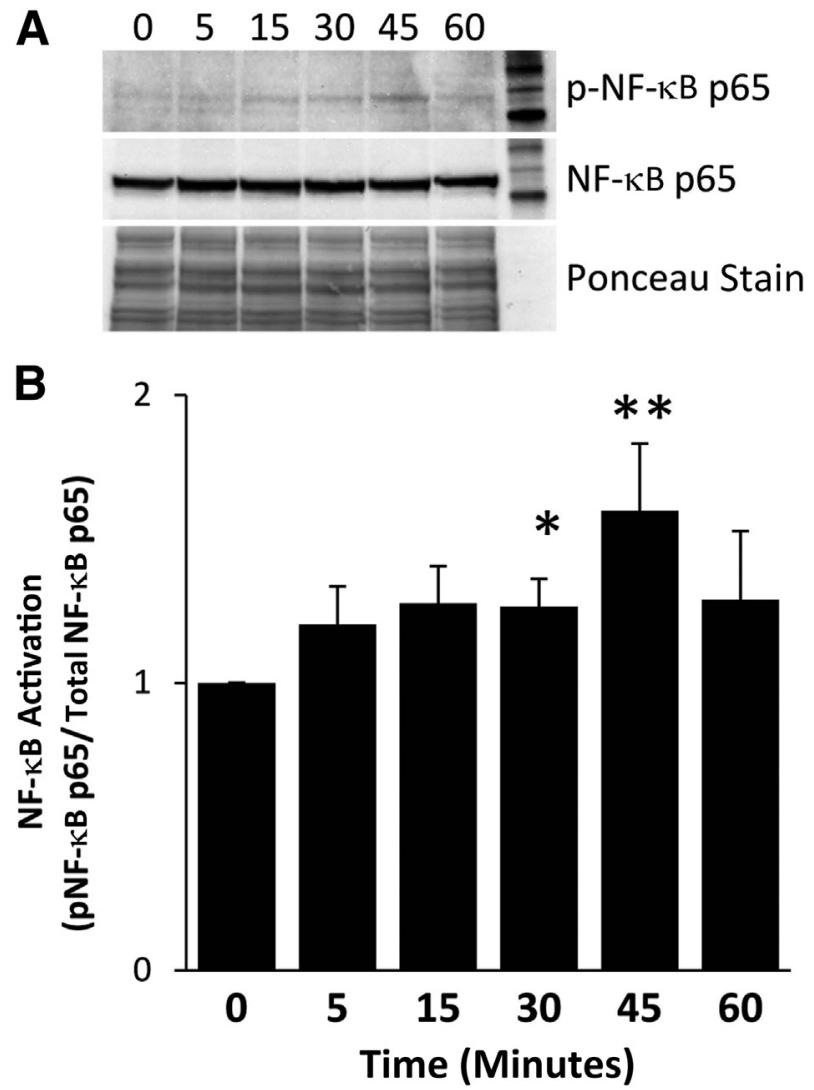

Figure 4 Tumor necrosis factor (TNF)- $\alpha$ activates NF- $\kappa B$ p65 in placental explants. Placental explants $(n=5, \mathbf{B})$ were incubated with 10 $\mathrm{ng} / \mathrm{mL}$ TNF- $\alpha$ for indicated time periods. A: Phosphorylated NF- $\kappa B$ p65 (p-NF-kB p65) and total NF- $\kappa B$ p65 were analyzed by Western blot analysis. Nitrocellulose membranes were stained with Ponceau $S$ solution to verify equal protein loading and blotting efficiency. B: Semiquantitative band analysis reveals peak activation of NF- $\kappa \mathrm{B}$ p 65 after 45-minute incubation with TNF- $\alpha$. Band densities of p-NF-kB p65 were related to corresponding densities of NF- $\kappa B$ p 65 and time 0 set as 1 . Values are given as means \pm SEM. ${ }^{*} P \leq 0.05,{ }^{*} P \leq 0.01$.

early-onset PE, compared to controls (Figure 1F). This discrepancy between results from ELISA and immunohistochemical quantification may be explained not only by the differences in method, but also by different antibodies used in these methods.

Significant differences in observed mRNA levels and the nonsignificant increase in protein levels measured by ELISA could be explained by the fact that membrane-bound fractalkine is cleaved by the metalloproteases ADAM10 and ADAM17, which have been described to release soluble fractalkine from the syncytiotrophoblast by metalloproteolytic shedding. Increased ADAM10 and ADAM17 may increase shedding of soluble fractalkine, resulting in decreased membrane-bound fractalkine. To consider this aspect, expression of ADAM10 and ADAM17 was analyzed. Accordingly, expression of both ADAM10 and ADAM17 was significantly increased $4.17 \pm 0.91$-fold and $2.43 \pm 0.30$-fold, respectively, in placentas from severe early-onset PE, when compared to controls (Figure 2).
Placental Fractalkine Is Up-Regulated by TNF- $\alpha$, But Not IL-6

Because PE has been suggested to have its origin in the first trimester of pregnancy and has been associated with a proinflammatory cytokine signature, including elevated maternal circulating IL-6 and TNF- $\alpha$ levels, ${ }^{26-28}$ we next tested the effect of these cytokines on fractalkine expression in first-trimester placental explants. Placental explants were incubated in the presence or absence of IL- 6 and TNF- $\alpha$ or the combination of both at 1 and $10 \mathrm{ng} / \mathrm{mL}$. Gene expression analysis revealed a significant $6.37 \pm 3.00$ - and $4.37 \pm$ 1.22-fold up-regulation of placental fractalkine in the presence of TNF- $\alpha$ alone and TNF- $\alpha$ in combination with IL-6, respectively, at a concentration of $10 \mathrm{ng} / \mathrm{mL}$ after 48 hours of treatment (Figure 3A). IL-6 alone did not affect placental fractalkine expression at both concentrations, which leads us to suggest that the observed effects of combined IL-6 and TNF- $\alpha$ treatment were only driven by TNF- $\alpha$. Analysis of the respective tissue protein and released soluble fractalkine
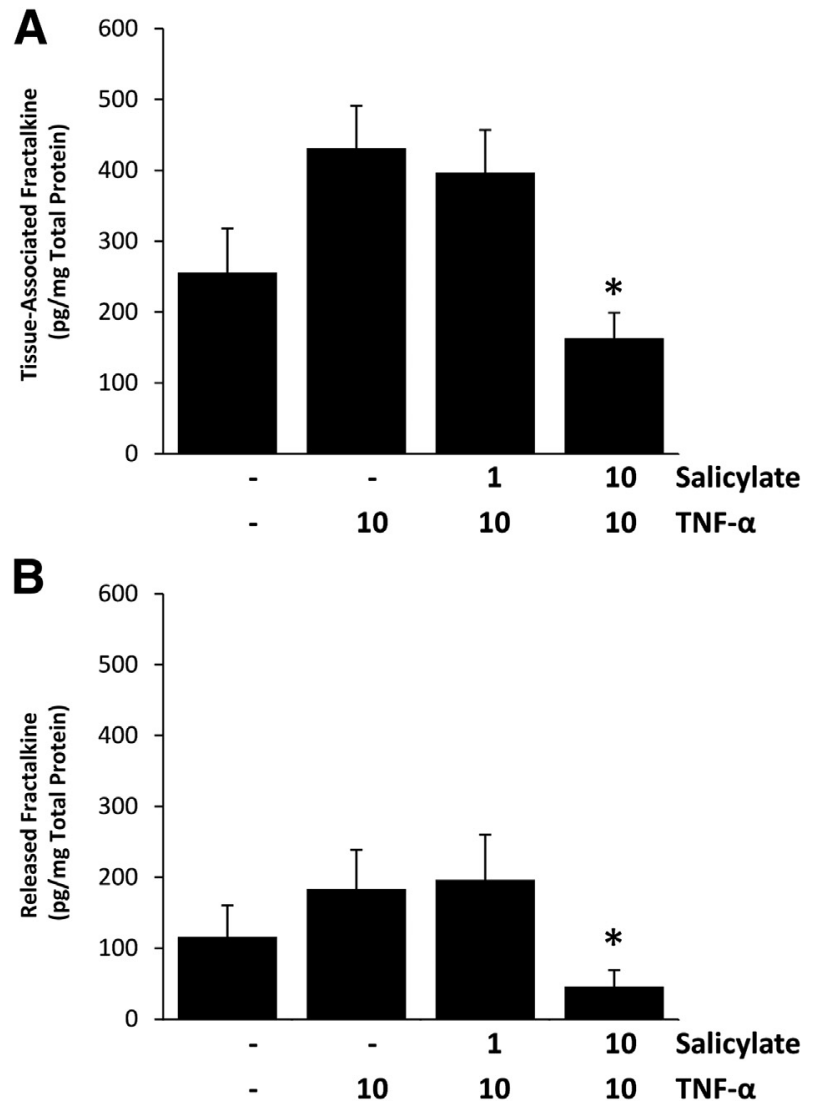

Figure 5 Salicylate reverses tumor necrosis factor (TNF)- $\alpha$-mediated up-regulation of placental fractalkine. Placental explants $(n=5)$ were incubated in the presence of $10 \mathrm{ng} / \mathrm{mL}$ TNF- $\alpha$ with or without salicylate at indicated concentrations for 48 hours. Enzyme-linked immunosorbent assay analysis of tissue homogenates (A) and culture supernatants (B) showed that salicylate at $10 \mu \mathrm{mol} / \mathrm{L}$, but not at $1 \mu \mathrm{mol} / \mathrm{L}$, significantly diminished TNF- $\alpha$-mediated up-regulation and release of placental fractalkine. Values are given as means \pm SEM. ${ }^{*} P \leq 0.05$. 
A

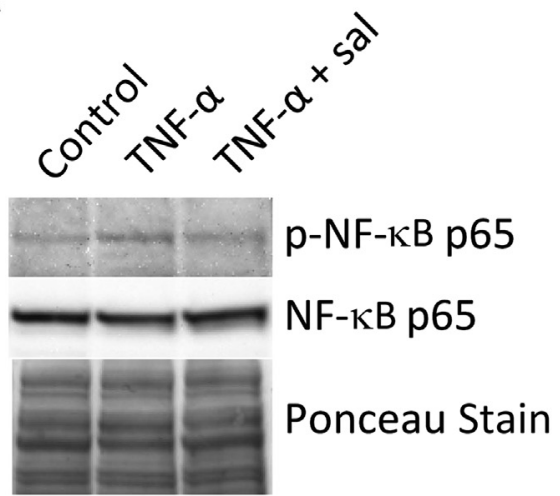

B

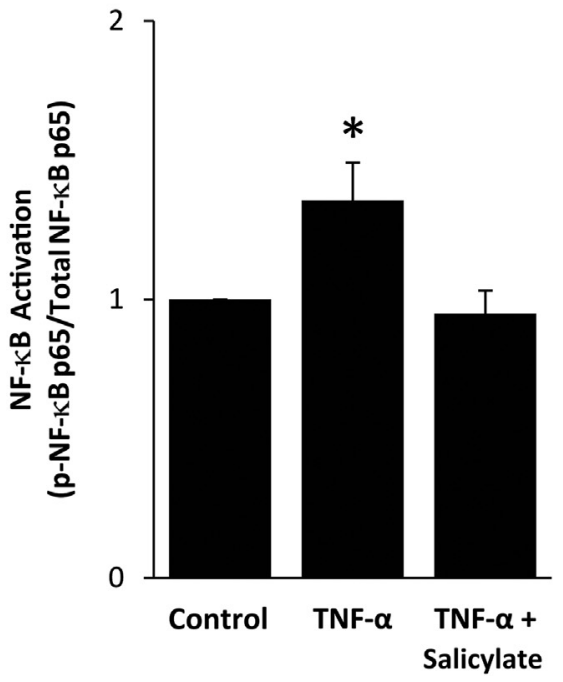

Figure 6 Salicylate impairs tumor necrosis factor (TNF)- $\alpha$-mediated activation of NF- $\kappa$ B p65 in placental explants. Placental explants $(n=5, \mathbf{B})$ were incubated in the presence of $10 \mathrm{ng} / \mathrm{mL} \mathrm{TNF}-\alpha$ with or without 10 $\mu \mathrm{mol} / \mathrm{L}$ salicylate for 45 minutes. A: Phosphorylated NF- $\kappa B$ p65 (p-NF-kB p65) and total NF- $\kappa B$ p65 were analyzed by Western blot analysis. Nitrocellulose membranes were stained with Ponceau $S$ solution to verify equal protein loading and blotting efficiency. B: Semiquantitative band analysis shows that salicylate significantly reduces TNF- $\alpha$-mediated phosphorylation of NF- $\kappa B$ p65. Band densities of p-NF-kB p65 were related to corresponding densities of NF-KB p65 and control set as 1 . Values are given as means \pm SEM. ${ }^{*} P \leq 0.05$.

confirmed mRNA data, albeit effects were less pronounced. Tissue-associated fractalkine protein increased $1.65 \pm 0.21$ and $1.79 \pm 0.19$-fold in the presence of $10 \mathrm{ng} / \mathrm{mL} \mathrm{TNF-} \alpha$ alone and TNF- $\alpha$ combined with IL-6, respectively, whereas no effects were detected in the presence of IL-6 alone (Figure 3B). Similar effects were detected in the supernatants of explant cultures, where released soluble fractalkine increased $1.81 \pm 0.24$ - and $2.17 \pm 0.39$-fold on treatment with $10 \mathrm{ng} / \mathrm{mL} \mathrm{TNF}-\alpha$ and TNF- $\alpha$ in combination with IL-6. Incubation of placental explants with IL-6 alone had no effects on the level of soluble fractalkine (Figure 3C). To determine any cytotoxic effects of TNF- $\alpha$ and IL-6 on placental explants, the release of $\mathrm{LDH}$ into the culture medium was analyzed after culture and showed no significant differences between treatments (data not shown).
TNF- $\alpha$-Induced Up-Regulation of Placental Fractalkine Is Mediated via NF- $\kappa$ B Pathway and Reversed by Salicylate

Accumulating experimental evidence suggests a role of the $\mathrm{NF}-\kappa \mathrm{B}$ pathway in the regulation of fractalkine expression in endothelial and epithelial cells..$^{29-31}$ On the basis of these findings, we next tested whether TNF- $\alpha$ treatment of placental explants results in activation of NF- $\kappa \mathrm{B}$. Incubation of placental explants with TNF- $\alpha$ over a period of 1 hour showed peak activation of $N F-\kappa B$ p65 after 45 minutes (Figure 4, A and B). To prove whether the activation of NF- $\kappa$ B p65 mediated up-regulation of placental fractalkine, placental explants were incubated with TNF- $\alpha$ in the presence or absence of salicylate, the basic derivative of aspirin, which recently has been suggested as a cheap, but nevertheless effective, drug to efficiently reverse TNF- $\alpha$-induced up-regulation of fractalkine through the NF- $\kappa$ B p65 pathway. ${ }^{32,33}$ Analysis of tissue-associated fractalkine in placental explants showed that salicylate at a concentration of $10 \mu \mathrm{mol} / \mathrm{L}$ significantly decreased TNF- $\alpha-$ mediated up-regulation of placental fractalkine by $62.2 \% \pm 8.4 \%$, whereas salicylate at $1 \mu \mathrm{mol} / \mathrm{L}$ did not show a significant effect after 48 hours of incubation (Figure 5A). Measurements of released soluble fractalkine in supernatants of treated explants followed the trend observed for tissue-associated fractalkine. Although salicylate had no effect at $1 \mu \mathrm{mol} / \mathrm{L}$, it significantly diminished TNF- $\alpha$-induced release of placental fractalkine by $75.2 \% \pm 12.8 \%$ after 48 hours of incubation at 10 $\mu \mathrm{mol} / \mathrm{L}$ (Figure 5B). To exclude any cytotoxic effects of salicylate or combined TNF- $\alpha$ and salicylate treatment on placental explants, the release of LDH into the culture medium was analyzed after culture and showed no significant difference between treatments (data not shown).

Finally, activation of NF- $\kappa \mathrm{B}$ p65 on TNF- $\alpha$ stimulation was compared in placental explants in the presence or absence of salicylate. When explants were incubated with $\mathrm{TNF}-\alpha$ alone, an increase in phosphorylated NF- $\kappa \mathrm{B}$ p 65 was detected after 45 minutes, whereas salicylate significantly reversed this effect to the control level (Figure 6, A and B).

\section{Discussion}

Although maternal circulating cytokines were not analyzed in the present study, data from placental explant experiments suggest that increased maternal circulating TNF- $\alpha$, but not IL-6, up-regulates the expression and release of placental fractalkine in PE. This up-regulation may be initiated early in pregnancy, because longitudinal studies detected elevated maternal circulating levels of TNF- $\alpha$ during the first trimester in women who were destined to develop PE compared to women destined to have a normotensive pregnancy. ${ }^{34,35}$ This assumption is in good agreement with our data from first-trimester placental 
explants showing increased expression and release of placental fractalkine in response to TNF- $\alpha$. Speculations about the consequences of up-regulated placental fractalkine, however, should include the dual nature of the chemokine, both acting as a transmembrane adhesion molecule and being a soluble factor.

Previous studies by Stepanian et $\mathrm{al}^{36}$ suggested a trend toward elevated levels of soluble fractalkine in patients with early-onset PE, which fits well with our results. However, it should clearly be stressed at this point that soluble fractalkine in the circulation of patients with early-onset PE may not only derive from placental villi, but also from endothelial cells of the maternal vasculature. Thus, elevated soluble fractalkine may derive from fetal and maternal origin. Contribution of placental fractalkine to the maternal pool of soluble fractalkine during gestation may be relatively low because the entire surface of placental villi at term, with approximately 12 to $15 \mathrm{~m}^{2}$, represents only a small area compared to approximately 4000 to $7000 \mathrm{~m}^{2}$ endothelium of maternal blood vessels. ${ }^{37,38}$ However, in the case of placental fractalkine, the function of the soluble form may comprise recruitment of CX3CR1-expressing monocytes, natural killer cells, and T-cell subsets to the fetal-maternal interface.

Our finding of increased placental ADAM10 and ADAM17 expression in severe early-onset PE confirms previous studies, which also demonstrated increased expression of both metalloproteases in preeclamptic placentas. ${ }^{39,40}$ Up-regulation of placental fractalkine, together with increased placental ADAM10 and ADAM17 expression, suggests enhanced shedding and release of placental fractalkine, which, in turn, may increase recruitment of maternal leukocytes in severe early-onset PE. This way, placental fractalkine could contribute to activation of maternal monocytes and type 1 helper T-cell lymphocytes, the predominant cell types mediating the inflammatory cascade in women with PE. ${ }^{41,42}$

Although not necessarily the primary cause of PE, leukocyte activation is suggested to contribute to the progression of this pregnancy pathology. ${ }^{43}$ Activation of leukocytes during their uteroplacental passage has been suggested by Mellembakken et al, ${ }^{43}$ who detected increased adhesion molecules and complement-related factors on neutrophils and monocytes in samples from uterine veins compared to samples from antecubital veins in patients with severe PE. Thus, activation of leukocytes by placental factors, such as placental fractalkine, may, in turn, initiate a vicious circle of leukocyte activation leading to a persistent, exaggerated, proinflammatory uteroplacental environment in PE. Whether this activation depends on direct cellular interaction of maternal leukocytes with the syncytiotrophoblast remains speculative. However, incubation of monocytes with soluble fractalkine has been shown to significantly up-regulate TNF- $\alpha$ expression, ${ }^{44}$ and rather argues for activation by soluble fractalkine independent of direct intercellular contact. On the basis of this concept, leukocyte-derived cytokines, such as TNF- $\alpha$, may sustain up-regulation of placental fractalkine expression in the syncytiotrophoblast.

Our placental explant experiments show activation of the NF- $\kappa \mathrm{B}$ pathway in response to TNF- $\alpha$, which is supported by previous studies with primary trophoblasts and trophoblast cell lines. ${ }^{45,46}$ Salicylate effectively inhibited TNF- $\alpha$-induced upregulation of placental fractalkine by interfering with NF- $\kappa \mathrm{B}$ activation in explant culture at a concentration of $10 \mu \mathrm{mol} / \mathrm{L}$. However, salicylate derivatives, such as aspirin, at suprapharmacological concentrations $(>5 \mathrm{mmol} / \mathrm{L})$ exhibit inhibitory actions on several transcriptional activators and, in fact, are considered as toxic for human subjects. ${ }^{47,48}$ Nevertheless, highdose aspirin treatment not only reduced the severity of atherosclerotic plaques, but also significantly reduced fractalkine expression in aortas of apolipoprotein E knockout mice. ${ }^{33} \mathrm{In}$ humans, low-dose aspirin treatment (50 to $150 \mathrm{mg}$ /day) of women at high risk for $\mathrm{PE}$ has been suggested to reduce the incidence and severity of PE without causing harm. Metaanalyses of randomized controlled studies revealed that low-dose aspirin treatment, when started $\leq 16$ weeks, significantly reduced the risk for severe PE. ${ }^{4 \overline{9}, 50}$ Whether low-dose aspirin treatment down-regulates placental fractalkine expression in severe PE remains open. However, direct interference with the fractalkine/CX3CR1 axis may be a more eligible strategy. Potent and selective orally available CX3CR1 antagonists have recently been developed and inhibited fractalkine-induced activation of CX3CR1 on blood monocytes in a rat model for multiple sclerosis. ${ }^{51,52}$

In summary, we conclude that TNF- $\alpha$ up-regulates placental fractalkine expression and secretion via activation of the NF- $\kappa$ B pathway. On the basis of our results, we propose the concept that up-regulated placental fractalkine, together with up-regulated placental ADAM10 and ADM17 expression, may lead to increased release of soluble fractalkine into the maternal circulation, where it may contribute to an exaggerated systemic inflammatory response in severe early-onset PE.

\section{Acknowledgments}

We thank Bettina Amtmann and Petra Wagner for recruiting donors for placental tissue samples for this study and Dr. Andreas Glasner for providing first-trimester placenta tissues.

\section{References}

1. Huppertz B: Placental origins of preeclampsia: challenging the current hypothesis. Hypertension 2008, 51:970-975

2. Lau SY, Guild SJ, Barrett CJ, Chen Q, McCowan L, Jordan V, Chamley LW: Tumor necrosis factor-alpha, interleukin-6, and interleukin-10 levels are altered in preeclampsia: a systematic review and meta-analysis. Am J Reprod Immunol 2013, 70:412-427

3. Chen LM, Liu B, Zhao HB, Stone P, Chen Q, Chamley L: IL-6, TNFalpha and TGFbeta promote nonapoptotic trophoblast deportation and subsequently causes endothelial cell activation. Placenta 2010, 31:75-80 
4. Goswami D, Tannetta DS, Magee LA, Fuchisawa A, Redman CW, Sargent IL, von Dadelszen P: Excess syncytiotrophoblast microparticle shedding is a feature of early-onset pre-eclampsia, but not normotensive intrauterine growth restriction. Placenta 2006, 27: $56-61$

5. Makris A, Thornton C, Thompson J, Thomson S, Martin R, Ogle R, Waugh R, McKenzie P, Kirwan P, Hennessy A: Uteroplacental ischemia results in proteinuric hypertension and elevated sFLT-1. Kidney Int 2007, 71:977-984

6. Siwetz M, Blaschitz A, Kremshofer J, Bilic J, Desoye G, Huppertz B, Gauster M: Metalloprotease dependent release of placenta derived fractalkine. Mediators Inflamm 2014, 2014:839290

7. Umehara H, Bloom ET, Okazaki T, Nagano Y, Yoshie O, Imai T: Fractalkine in vascular biology: from basic research to clinical disease. Arterioscler Thromb Vasc Biol 2004, 24:34-40

8. Jones BA, Beamer M, Ahmed S: Fractalkine/CX3CL1: a potential new target for inflammatory diseases. Mol Interv 2010, 10: $263-270$

9. Ludwig A, Weber C: Transmembrane chemokines: versatile 'special agents' in vascular inflammation. Thromb Haemost 2007, 97: 694-703

10. Garton KJ, Gough PJ, Blobel CP, Murphy G, Greaves DR, Dempsey PJ, Raines EW: Tumor necrosis factor-alpha-converting enzyme (ADAM17) mediates the cleavage and shedding of fractalkine (CX3CL1). J Biol Chem 2001, 276:37993-38001

11. Hundhausen C, Misztela D, Berkhout TA, Broadway N, Saftig P, Reiss K, Hartmann D, Fahrenholz F, Postina R, Matthews V, Kallen KJ, Rose-John S, Ludwig A: The disintegrin-like metalloproteinase ADAM10 is involved in constitutive cleavage of CX3CL1 (fractalkine) and regulates CX3CL1-mediated cell-cell adhesion. Blood 2003, 102:1186-1195

12. Hundhausen C, Schulte A, Schulz B, Andrzejewski MG, Schwarz N, von Hundelshausen P, Winter U, Paliga K, Reiss K, Saftig P, Weber C, Ludwig A: Regulated shedding of transmembrane chemokines by the disintegrin and metalloproteinase 10 facilitates detachment of adherent leukocytes. J Immunol 2007, 178:8064-8072

13. Imai T, Hieshima K, Haskell C, Baba M, Nagira M, Nishimura M, Kakizaki M, Takagi S, Nomiyama H, Schall TJ, Yoshie O: Identification and molecular characterization of fractalkine receptor CX3CR1, which mediates both leukocyte migration and adhesion. Cell 1997, 91:521-530

14. D'Haese JG, Demir IE, Friess H, Ceyhan GO: Fractalkine/CX3CR1: why a single chemokine-receptor duo bears a major and unique therapeutic potential. Expert Opin Ther Targets 2010, $14: 207-219$

15. Cammas L, Reinaud P, Dubois O, Bordas N, Germain G, Charpigny G: Identification of differentially regulated genes during elongation and early implantation in the ovine trophoblast using complementary DNA array screening. Biol Reprod 2005, 72: 960-967

16. Hannan NJ, Jones RL, White CA, Salamonsen LA: The chemokines, CX3CL1, CCL14, and CCL4, promote human trophoblast migration at the feto-maternal interface. Biol Reprod 2006, 74:896-904

17. Hannan NJ, Salamonsen LA: CX3CL1 and CCL14 regulate extracellular matrix and adhesion molecules in the trophoblast: potential roles in human embryo implantation. Biol Reprod 2008, 79:58-65

18. Szukiewicz D, Kochanowski J, Pyzlak M, Szewczyk G, Stangret A, Mittal TK: Fractalkine (CX3CL1) and its receptor CX3CR1 may contribute to increased angiogenesis in diabetic placenta. Mediators Inflamm 2013, 2013:437576

19. Szukiewicz D, Kochanowski J, Mittal TK, Pyzlak M, Szewczyk G, Cendrowski K: CX3CL1 (fractalkine) and TNFalpha production by perfused human placental lobules under normoxic and hypoxic conditions in vitro: the importance of CX3CR1 signaling. Inflamm Res 2014, 63:179-189

20. Szukiewicz D, Kochanowski J, Mittal TK, Pyzlak M, Szewczyk G, Cendrowski K: Chorioamnionitis (ChA) modifies CX3CL1 (fractalkine) production by human amniotic epithelial cells (HAEC) under normoxic and hypoxic conditions. J Inflamm (Lond) 2014, 11:12

21. Hauguel-de Mouzon S, Guerre-Millo M: The placenta cytokine network and inflammatory signals. Placenta 2006, 27:794-798

22. Borzychowski AM, Sargent IL, Redman CW: Inflammation and preeclampsia. Semin Fetal Neonatal Med 2006, 11:309-316

23. Sacks GP, Studena K, Sargent K, Redman CW: Normal pregnancy and preeclampsia both produce inflammatory changes in peripheral blood leukocytes akin to those of sepsis. Am J Obstet Gynecol 1998, 179:80-86

24. Austgulen R, Lien E, Liabakk NB, Jacobsen G, Arntzen KJ: Increased levels of cytokines and cytokine activity modifiers in normal pregnancy. Eur J Obstet Gynecol Reprod Biol 1994, 57: 149-155

25. Sommer C, Strähle C, Köthe U, Hamprecht FA: ilastik: Interactive Learning and Segmentation Toolkit. Eighth IEEE International Symposium on Biomedical Imaging (ISBI) Proceedings, (2011), 230233

26. Pinheiro MB, Martins-Filho OA, Mota AP, Alpoim PN, Godoi LC, Silveira AC, Teixeira-Carvalho A, Gomes KB, Dusse LM: Severe preeclampsia goes along with a cytokine network disturbance towards a systemic inflammatory state. Cytokine 2013, 62:165-173

27. Tosun M, Celik H, Avci B, Yavuz E, Alper T, Malatyalioglu E: Maternal and umbilical serum levels of interleukin-6, interleukin-8, and tumor necrosis factor-alpha in normal pregnancies and in pregnancies complicated by preeclampsia. J Matern Fetal Neonatal Med 2010, 23:880-886

28. Guven MA, Coskun A, Ertas IE, Aral M, Zencirci B, Oksuz H: Association of maternal serum CRP, IL-6, TNF-alpha, homocysteine, folic acid and vitamin B12 levels with the severity of preeclampsia and fetal birth weight. Hypertens Pregnancy 2009, 28:190-200

29. Ahn SY, Cho CH, Park KG, Lee HJ, Lee S, Park SK, Lee IK, Koh GY: Tumor necrosis factor-alpha induces fractalkine expression preferentially in arterial endothelial cells and mithramycin A suppresses TNF-alpha-induced fractalkine expression. Am J Pathol 2004, $164: 1663-1672$

30. Bhavsar PK, Sukkar MB, Khorasani N, Lee KY, Chung KF: Glucocorticoid suppression of CX3CL1 (fractalkine) by reduced gene promoter recruitment of NF-kappaB. FASEB J 2008, 22:1807-1816

31. Chen T, Guo ZP, Jiao XY, Jia RZ, Zhang YH, Li JY, Huang XL, Liu HJ: Peoniflorin suppresses tumor necrosis factor-alpha induced chemokine production in human dermal microvascular endothelial cells by blocking nuclear factor-kappaB and ERK pathway. Arch Dermatol Res 2011, 303:351-360

32. Jiang DQ, Liu H, Zhang SB, Zhang XL: Aspirin inhibits tumor necrosis factor-alpha-stimulated fractalkine expression in human umbilical vein endothelial cells. Chin Med J (Engl) 2009, 122: $1147-1153$

33. Liu H, Jiang D, Zhang S, Ou B: Aspirin inhibits fractalkine expression in atherosclerotic plaques and reduces atherosclerosis in ApoE gene knockout mice. Cardiovasc Drugs Ther 2010, 24:17-24

34. Omu AE, Al-Qattan F, Diejomaoh ME, Al-Yatama M: Differential levels of $\mathrm{T}$ helper cytokines in preeclampsia: pregnancy, labor and puerperium. Acta Obstet Gynecol Scand 1999, 78:675-680

35. Hamai Y, Fujii T, Yamashita T, Nishina H, Kozuma S, Mikami Y, Taketani Y: Evidence for an elevation in serum interleukin-2 and tumor necrosis factor-alpha levels before the clinical manifestations of preeclampsia. Am J Reprod Immunol 1997, 38:89-93

36. Stepanian A, Benchenni S, Beillat-Lucas T, Omnes S, Defay F, Peynaud-Debayle E, Baron G, Le Querrec A, Dreyfus M, Salomon L, Tsatsaris V, de Prost D, Mandelbrot L; ECLAXIR Study Group: Search for an association between V249I and T280M CX3CR1 genetic polymorphisms, endothelial injury and preeclampsia: the ECLAXIR study. PLoS One 2009, 4:e6192

37. Benirschke K, Kaufmann P, Baergen RN: Pathology of the Human Placenta. Edited by Anonymous: New York,, Springer, 2006, pp $42-49$ 
38. Aird WC: Spatial and temporal dynamics of the endothelium. J Thromb Haemost 2005, 3:1392-1406

39. Zhao S, Gu Y, Fan R, Groome LJ, Cooper D, Wang Y: Proteases and sFlt-1 release in the human placenta. Placenta 2010, 31:512-518

40. Yang Y, Wang Y, Zeng X, Ma XJ, Zhao Y, Qiao J, Cao B, Li YX, Ji L, Wang YL: Self-control of HGF regulation on human trophoblast cell invasion via enhancing c-Met receptor shedding by ADAM10 and ADAM17. J Clin Endocrinol Metab 2012, 97: E1390-E1401

41. Sharma A, Satyam A, Sharma JB: Leptin, IL-10 and inflammatory markers (TNF-alpha, IL-6 and IL-8) in pre-eclamptic, normotensive pregnant and healthy non-pregnant women. Am J Reprod Immunol 2007, 58:21-30

42. Conrad KP, Benyo DF: Placental cytokines and the pathogenesis of preeclampsia. Am J Reprod Immunol 1997, 37:240-249

43. Mellembakken JR, Aukrust P, Olafsen MK, Ueland T, Hestdal K, Videm V: Activation of leukocytes during the uteroplacental passage in preeclampsia. Hypertension 2002, 39:155-160

44. Butoi ED, Gan AM, Manduteanu I, Stan D, Calin M, Pirvulescu M, Koenen RR, Weber C, Simionescu M: Cross talk between smooth muscle cells and monocytes/activated monocytes via CX3CL1/CX3CR1 axis augments expression of pro-atherogenic molecules. Biochim Biophys Acta 2011, 1813:2026-2035

45. Li W, Li H, Bocking AD, Challis JR: Tumor necrosis factor stimulates matrix metalloproteinase 9 secretion from cultured human chorionic trophoblast cells through TNF receptor 1 signaling to IKBKB-NFKB and MAPK1/3 pathway. Biol Reprod 2010, 83: $481-487$
46. Huber AV, Saleh L, Bauer S, Husslein P, Knofler M: TNFalphamediated induction of PAI-1 restricts invasion of HTR-8/SVneo trophoblast cells. Placenta 2006, 27:127-136

47. Frantz B, O'Neill EA: The effect of sodium salicylate and aspirin on NF-kappa B. Science 1995, 270:2017-2019

48. Wu KK: Aspirin and salicylate: an old remedy with a new twist. Circulation 2000, 102:2022-2023

49. Roberge S, Giguere Y, Villa P, Nicolaides K, Vainio M, Forest JC, von Dadelszen P, Vaiman D, Tapp S, Bujold E: Early administration of low-dose aspirin for the prevention of severe and mild preeclampsia: a systematic review and meta-analysis. Am J Perinatol 2012, 29:551-556

50. Bujold E, Morency AM, Roberge S, Lacasse Y, Forest JC, Giguere Y: Acetylsalicylic acid for the prevention of preeclampsia and intra-uterine growth restriction in women with abnormal uterine artery Doppler: a systematic review and meta-analysis. J Obstet Gynaecol Can 2009, 31:818-826

51. Karlstrom S, Nordvall G, Sohn D, Hettman A, Turek D, Ahlin K, Kers A, Claesson M, Slivo C, Lo-Alfredsson Y, Petersson C, Bessidskaia G, Svensson PH, Rein T, Jerning E, Malmberg A, Ahlgen C, Ray C, Vares L, Ivanov V, Johansson R: Substituted 7-amino-5-thio-thiazolo [4,5-d]pyrimidines as potent and selective antagonists of the fractalkine receptor (CX3CR1). J Med Chem 2013, 56:3177-3190

52. Ridderstad Wollberg A, Ericsson-Dahlstrand A, Jureus A, Ekerot P, Simon S, Nilsson M, Wiklund SJ, Berg AL, Ferm M, Sunnemark D, Johansson R: Pharmacological inhibition of the chemokine receptor CX3CR1 attenuates disease in a chronic-relapsing rat model for multiple sclerosis. Proc Natl Acad Sci U S A 2014, 111:5409-5414 